P. Griffith

Nagoya Math. J.

Vol. 72 (1978), $71-82$

\title{
COMPLETIONS OF RINGS OF INVARIANTS AND THEIR DIVISOR CLASS GROUPS
}

\author{
PHILLIP GRIFFITH
}

\section{Introduction}

Let $k$ be a field and let $A=\amalg_{i \geq 0} A_{i}$ be a normal graded subring of the full ring of polynomials $R=k\left[X_{1}, \cdots, X_{n}\right]$ (where $R$ always is graded via total degree and $A_{0}=k$ ). R. Fossum and the author [F-G] observed that the completion $\hat{A}$ at the irrelevant maximal ideal of $A$ is isomorphic to the subring $\prod_{i \geq 0} A_{i}$ of the formal power series ring $\hat{R}$ $=k\left[\left[X_{1}, \ldots, X_{n}\right]\right]$ and, moreover, that $\hat{A}$ is a ring of invariants of an algebraic group whenever $A$ is. In the aforementioned paper, the authors needed to know that $\hat{A}$ remained a factorial ring in case $k$ was of characteristic $p>0$ and $A$ was the ring of invariants of $Z / p^{n} Z$, where the action of $\boldsymbol{Z} / p^{n} \boldsymbol{Z}$ was induced from the regular representation on a $k$-vector space of dimension $p^{n}$. Having settled this question in the affirmative, R. Fossum and the author were able to obtain many examples of non Cohen-Macaulay, factorial domains as well as show that the completion of M. Bertin's example [B] was also non Cohen-Macaulay and factorial. Rather difficult computations in group cohomology were required in order to establish the factoriality of $\hat{A}$ in these cases. In the same vein Danilov [D] has shown using geometric arguments that, if $A$ is a graded ring which is generated by its 1-forms and if $\operatorname{Proj}(A)$ is smooth over $k$, then the map of divisor class groups $C \ell(A) \rightarrow C \ell(\hat{A})$ is an isomorphism provided certain cohomology groups vanish. One negative note on the matter is that the map $C \ell(A) \rightarrow C \ell(\hat{A})$ need not be an isomorphism when $A$ is a ring of invariants of derivations in positive characteristics (see [F; pp. 101, 102]).

The situation we wish to discuss in this paper goes as follows. Let $V$ be an $n$-dimensional $k$-vector space over an algebraically closed field

Received June 27, 1977.

This research was partially supported by the National Science Foundation. 
$k$ and suppose the linearly reductive, affine algebraic group $G$ has a linear representation $\rho: G \rightarrow G L(V)$. This representation induces an action of $G$ on $R=k\left[X_{1}, \cdots, X_{n}\right]$, which preserves the natural grading on $R$. So $A=R^{G}$ is (in this case) a finitely generated $k$-algebra as well as a graded subring of $R$. Our main goal is to show that the natural map of divisor class groups $C \ell(A) \rightarrow C \ell(\hat{A})$ is an isomorphism. The more general case concerning reductive groups is not yet resolved, although $\mathrm{R}$. Fossum has communicated some (unpublished) results in that direction. We would like to thank M. Hochster for pointing out a mistake in an earlier draft.

\section{Some general facts about groups of units, divisor class groups and cohomology}

As a general reference for this section one may consult Fossum's book [F; Chapters IV and V]. In addition to the notation cited in our introduction, we shall generally use $G_{m}(B)$ to denote the multiplicative group of units of a ring $B$, although we shall deviate from this in the case of fields and use the usual notation of $k^{*}$. One should also recall that the divisor class group of a normal domain $B$ (denoted $C \ell(B)$ ) is the set of isomorphism classes of divisorial (i.e. reflexive) ideals of $B$ together with the operation: $\left.[\mathfrak{a}] \cdot[\mathfrak{b}]=\left[\operatorname{Hom}\left(\mathfrak{a}^{*}, \mathfrak{b}\right)\right]=[\mathfrak{a} \otimes \mathfrak{b})^{* *}\right]$, where $\mathfrak{a}$ and $\mathfrak{b}$ are reflexive ideals and $\mathfrak{a}^{*}=\operatorname{Hom}(\mathfrak{a}, B)$. Unless otherwise stated, all graded normal domains $B$ will be finitely generated over $B_{0}$ $=k$, where $k$ is a field.

LEMMA 1.1. Let the normal domain $B$ be a finitely generated, graded k-algebra and let $G$ be a linearly reductive affine algebraic group acting k-linearly on $B$. Suppose that $u: G \rightarrow G_{m}(\hat{B})$ is a one-cocycle for the induced action of $G$ on $\hat{B}$, that is, there is a nonzero $f \in \hat{B}$ for which $g(f)=u(g) f$, for each $g \in G$. Then there is a character $\chi$ of $G$ and a trivial one-cocycle $v: G \rightarrow G_{m}(\hat{B})$ i.e. $v(g)=\omega g\left(\omega^{-1}\right)$ for some fixed $\left.\omega \in G_{m}(\hat{R})\right)$ such that

$$
u(g)=\chi(g) v(g) \quad \text { for } g \in G
$$

Proof. Let $B_{(i)}$ denote the ith graded part of $B$. We can write the function $u=\sum_{i \geq 0} u_{i}$, where $u_{i}(g)$ is the homogeneous component of $u(g)$ in $B_{(i)}$. Since $B_{(0)}=k$ and since $k$ is $G$-trivial, the one cocycle 
condition on $u$ implies that $u_{0}: G \rightarrow k^{*}$ is a character. Put $\chi=u_{0}$ and set $v(g)=\chi^{-1}(g) u(g)$. Writing $v=\sum_{i \geq 0} v_{i}$ as $u$ was also written, one may easily observe that $v$ is a one-cocycle and that $v_{0} \equiv 1$.

For the moment let $w=\sum w_{i}$ be any one-cocycle (as above) with $w_{0} \equiv 1$. Let $n$ be the first positive integer such that $w_{n} \not \equiv 0$. The two conditions $w_{0} \equiv 1$ and $w(g h)=g(w(h)) w(g)$ give that $w_{n}(g h)=g\left(w_{n}(h)\right)$ $+w_{n}(g)$, that is, $w_{n}$ represents a one-cocycle in the Hochschild cohomology group ${\underset{\sim}{H}}^{1}\left(G, B_{(n)}\right)$. However, ${\underset{\sim}{H}}^{1}\left(G, B_{(n)}\right)=0$ since $G$ is linearly reductive (c.f. [D-G; page 194]). We therefore obtain an element $z \in B_{(n)}$ so that $w_{n}(g)=-z+g(z)$ for each $g \in G$. Now define the trivial multiplicative one-cocycle

$$
\lambda^{(n)}: G \rightarrow G_{m}(\hat{B})
$$

by $\lambda^{(n)}(g)=(1-z)^{-1} g(1-z)$, and further consider the new multiplicative one cocycle $w \lambda^{(n)}$. For $g \in G$ we have the expression

$$
w \lambda^{(n)}(g)=\left(\sum w_{i}(g)\right)\left(\sum z^{i}\right) g(1-z)
$$

Clearly the degree zero part of this expression is 1 and the terms of degree $i, i<n$, are zero since $w_{i} \equiv 0$, for $0<i<n$, and degree $z$ $=$ degree $g(z)=n$. Moreover, the term of degree $n$ is $\left(w_{n}(g)+z\right)-g(z)$. However, this term is also zero from the preceding discussion. Hence our "new $w$ " has at least one more positive degree term which is identically zero.

We now return to consider the one-cocycle $v: G \rightarrow G_{m}(\hat{B})$. By successively applying the above procedure, we obtain $z_{i} \in B_{(i)}$, for each $i>0$, and $\lambda^{(i)}: G \rightarrow G_{m}(\hat{B})$ defined by $\lambda^{(i)}(g)=\left(1-z_{i}\right)^{-1} g\left(1-z_{i}\right)$ so that $v \lambda^{(1)} \cdots \lambda^{(n)}(g)$ has no nonzero positive degree terms for $0<i \leq n$. Clearly the sequence of functions $\left\{\lambda^{(1)} \cdots \lambda^{(n)}\right\}_{n}$ converges to $u^{-1}$ in the m-adic topology ( $m$ is the irrelevant maximal ideal of $B$ ). Since degree $z_{i}=i$, it also follows that the sequence of units $\left(1-z_{1}\right) \cdots\left(1-z_{n}\right)$ converges to some unit $w$ in $\hat{B}$. Consequently we have that

$$
v^{-1}(g)=\lim \left(1-z_{1}\right)^{-1} g\left(1-z_{1}\right) \cdots\left(1-z_{n}\right)^{-1} g\left(1-z_{n}\right)
$$

or that $v^{-1}(g)=w^{-1} g(w)$. Hence $v(g)=w g\left(w^{-1}\right)$ for each $g \in G$. This completes our proof of Lemma 1.1.

LEMMA 1.2. Assume the setting is as in Lemma 1.1 together with 
the additional assumptions that $G$ is connected and that $\hat{B}$ is factorial (consequently $B$ is also factorial). If $f \in \hat{A}=\hat{B}^{G}$ divides a nonzero homogeneous element in $A=B^{G}$, then $f$ is an associate in $\hat{A}$ of a homogeneous element of $A$.

Proof. Suppose that $h$ is a homogeneous element of $A$ and that $f \in \hat{A}$ is such that $h=f d$. Since $\hat{B}$ is factorial and since homogeneous primes in $B$ necessarily remain primes in $\hat{B}$, it follows that $f$ is an associate in $\hat{B}$ of a homogeneous element, say $f=u w$, where $u \in G_{m}(\hat{B})$ and $w$ is homogeneous in $B$. Since $f$ is an invariant, it follows that the orbit of $w$ under $G$ is finite (up to associates). Therefore, the fact that $G$ is connected gives that $g(w)=u(g) w$, where $u(g) \in G_{m}(\hat{B})$. One easily checks from the multiplication that $u: G \rightarrow G_{m}(\hat{B})$ is a one-cocycle. Moreover, since $w$ is homogeneous, it follows that $u(g)$ is of degree zero for each $g$ and hence that $u$ is a character $u: G \rightarrow k^{*}$. After noticing that the degree zero term of $u$ is nonzero and invariant and that, of course, each homogeneous component of $f$ is invariant, then one may observe that $u$ is the trivial character. So $g(w)=w$ for each $g \in G$, and $w \in \hat{A}$; thus $u$ is necessarily also in $\hat{A}$. Thus, $f$ is an associate in $\hat{A}$ of a homogeneous element of $A$.

LEMMA 1.3. Let $k$ be an algebraically closed field and let $B$ be a normal domain that is also a graded k-algebra. Then there is an exact sequence of groups

$$
1 \rightarrow G_{m}(B) \rightarrow G_{m}(\hat{B}) \rightarrow D \rightarrow 1
$$

where:

a) $D$ is a torsion free abelian group that is divisible by every positive integer that is not divisible by the characteristic of $k$. (Hence, if char $k=0$, then $D$ is a divisible group).

b) If $\hat{B}$ is factorial or if $B=R^{G}$, where $R=k\left[T_{1}, \cdots, T_{n}\right]$ and $G$ is a connected affine algebraic group acting linearly on $R$, then for any nonzero homogeneous form $h$ in $B$ there is a natural isomorphism

$$
D \stackrel{\sim}{\longrightarrow} \operatorname{coker}\left(G_{m}\left(B\left[h^{-1}\right]\right) \rightarrow G_{m}\left(\hat{B}\left[h^{-1}\right]\right)\right) .
$$

Proof. Since $B$ is of finite type over a field and normal, it follows that $\hat{B}$ is a normal local domain and that an equation of the form 
$X^{n}-u=0, u \in G_{m}(B)$, having a solution in $\hat{B}$ necessarily has a solution in $B$. Hence $G_{m}(B)$ is a pure subgroup of $G_{m}(\hat{B})$. Since $k^{*}$ is contained in $G_{m}(B)$ and since $k$ is algebraically closed, it easily follows that all torsion elements of $G_{m}(\hat{B})$ are in $k^{*} \subseteq G_{m}(B)$. Therefore $D$ is torsion free. The statement in part a) concerning the divisibility of $D$ is a direct consequence of Hensel's Lemma and the lifting of factorizations from $k^{*}$ to $\hat{B}$ of polynomials of the type $X^{n}-u$.

In order to see part b) of Lemma 1.3, we first note that under either hypothesis on $B$ that, if $f \in \hat{B}$ divides a nonzero homogeneous form in $B$, then (from Lemma 1.2) $f=u h_{1}$, where $u \in G_{m}(\hat{B})$ and $h_{1}$ is homogeneous in $B$. Hence, if $f$ is a unit in $G_{m}\left(\hat{B}\left[h^{-1}\right]\right)$, then $f$ divides $h^{s}$ for some $s \geq 0$. So from the preceding discussion we have that $f=u h_{1}$, where $u \in G_{m}(\hat{B})$ and $h_{1}$ is a homogeneous element of $B$ which (necessarily) divides $h^{s}$. Consequently, the coset $f \cdot G_{m}\left(B\left[h^{-1}\right]\right)=u$ - $G_{m}\left(B\left[h^{-1}\right]\right)$ from which it follows that the natural map $D \rightarrow$ coker $\cdot\left[G_{m}\left(B\left[h^{-1}\right]\right) \rightarrow G_{m}\left(\hat{B}\left[h^{-1}\right]\right)\right]$ is an epimorphism. It is elementary to show that this map is monic.

Remark. For $k$ algebraically closed and $B=R=k\left[T_{1}, \ldots, T_{n}\right]$ there is an abelian group decomposition $G_{m}(\hat{R})=k^{*} \times D$, where $D$ $=\{u \in \hat{R} \mid u \equiv 1 \bmod \hat{\mathfrak{m}}\}$ and where $\hat{\mathfrak{m}}$ denotes the completion of the irrelevant maximal ideal. If $G$ operates on $R$ as described in the introduction (and consequently $G$ operates on $G_{m}(\hat{R})$ ), then the above decomposition is also a $G$-decomposition. Finally, when char $k=p>0$, then $D$ is a torsion free $p$-adic module which is complete and separated in the $p$-adic topology.

COROLLARY 1.4. Let $k$ be an algebraically closed field and let $G$ be a finite group with char $k$ not dividing the order of $G$ and let $G$ act linearly on the graded normal domain $B$. Let $A=B^{G}$. If $\hat{B}$ is factorial and if $A \rightarrow B$ is separable at all prime ideals of $A$ of height one, then we have natural isomorphisms

$$
H^{1}\left(G, k^{*}\right)=\operatorname{Hom}\left(G, k^{*}\right) \stackrel{\sim}{\longrightarrow} C \ell(A) \stackrel{\sim}{\longrightarrow} C \ell(\hat{A}) .
$$

Proof. Since the exact sequence $1 \rightarrow G_{m}(B) \rightarrow G_{m}(\hat{B}) \rightarrow D \rightarrow 1$ is easily seen to be a $G$-exact sequence and since $G$ is finite with $D$ torsion free and divisible by every prime dividing $|G|$, it follows that $H^{i}(G, D)=0$ 
for all $i>0$ and that $H^{0}\left(G, G_{m}(\hat{B})\right) \rightarrow H^{0}(G, D)$ is an epimorphism. So

$$
H^{1}\left(G, G_{m}(B)\right) \rightarrow H^{1}\left(G, G_{m}(\hat{B})\right)
$$

is an isomorphism. Moreover, $G_{m}(B)=k^{*}$ and, of course, $G$ acts trivially on $k^{*}$. Hence Galois descent and the commutative diagram

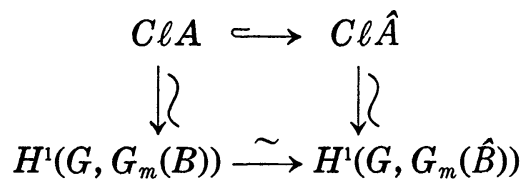

give the remaining conclusions. The isomorphisms of the divisor class groups of $A$ and $\hat{A}$ with the respective cohomology groups is a consequence of separability at prime ideals of height one (c.f. [F ; page 82]).

In the remaining results of this section, we show the importance of $\hat{B}$-divisorial ideals that are generated by homogeneous elements of $B$ in examining the map $C \ell B \rightarrow C \ell \hat{B}$.

LemMa 1.5. Let $B$ be a graded normal domain over the field $k$. If $\mathfrak{p}$ is a prime ideal of $\hat{B}$ and if $\mathfrak{q}$ is the $\hat{B}$-ideal, which is generated by all homogeneous elements of $\mathfrak{p}$, then $\mathfrak{q}$ is a prime ideal in $\hat{B}$ as well as being the completion of the prime ideal $q \cap B$.

Proof. Since $q$ has a generating set from $B$, it is clear that $\mathfrak{q} \cong \hat{B} \otimes_{B}(\mathfrak{q} \cap B)$. Moreover, since $\mathfrak{q} \cap B$ is a graded ideal $\mathfrak{q} \cap B=\mathfrak{a}$ $=\amalg_{i>0} a_{i}$ and since the product topology on graded modules agrees with the irrelevant maximal ideal topology (c.f. [EGA II, 2.1, 6 (vi)]), it follows that $\mathfrak{q}=\mathfrak{a}=\prod_{i>0} a_{i}$. Now suppose that $s, t \in \hat{B}$ and that $s \cdot t \in \mathfrak{q}$. But then the homogeneous components of $s \cdot t$ are in $q$. Then using the same elementary argument as in [F; page 41], we see that one of $s$ or $t$ has all of its components in $q$. Thus, by completeness of $q$, it now follows that $s \in \mathfrak{q}$ or $t \in \mathfrak{q}$.

As a consequence of this observation, we obtain a criterion for the divisor class group map $C \ell(B) \rightarrow C \ell(\hat{B})$ to be a bijection.

COROLLARY 1.6. Let $k$ be a field and $B$ a normal domain which is a graded k-algebra. Then there is an exact sequence

$$
0 \rightarrow C \ell(B) \rightarrow C \ell(\hat{B}) \rightarrow \operatorname{Pic}\left(S^{-1} \hat{B}\right) \rightarrow 0,
$$

where $S$ denotes the multiplicative semigroup of all nonzero homogeneous 
elements of $B$.

Proof. Suppose that some isomorphism class of an element in $C \ell(\hat{B})$ contains a divisorial ideal a with $\mathfrak{a} \cap S \neq \phi$. From Lemma 1.5, it follows that each prime ideal of height one in $\hat{B}$ which contains $a$ is the completion of a homogeneous prime ideal from $B$. So $\mathfrak{a}=\mathfrak{p}_{1}^{\left(e_{1}\right)} \cap \cdots \cap \mathfrak{p}_{t}^{\left(e_{t}\right)}$, where each $\mathfrak{p}_{i}$ is in the image of the map $C \ell B \rightarrow C \ell \hat{B}$. Thus, a must also be in the image of this map. It now follows that there is an exact sequence

$$
0 \rightarrow C \ell B \rightarrow C \ell \hat{B} \rightarrow C \ell\left(S^{-1} \hat{B}\right) \rightarrow 0 \text {. }
$$

But $C \ell\left(S^{-1} \hat{B}\right) \cong \operatorname{Pic}\left(S^{-1} \hat{B}\right)$, since $S^{-1} \hat{B}$ is necessarily locally regular. (Recall that $B \rightarrow \hat{B}$ has regular fibers and that all prime ideals in Spec $\left(S^{-1} \hat{B}\right)$ contract to regular prime ideals in $\left.B\right)$.

We now wish to rid ourselves of the apparant problem of separability in Corollary 1.4 .

Proposition 1.7. Let $k$ be an algebraically closed field and let $G$ be a finite group with $|G|$ not divisible by char $k$. Let $B$ be a graded normal domain over $k$ and suppose that $G$ acts linearly on $B$. Let $A$ $=B^{G}$. Suppose $B$ satisfies:

i) $\hat{B}\left[h_{1}^{-1}\right]$ is a UFD for some nonzero homogeneous element $h_{1}$ in $B$.

ii) The natural map coker $\left[G_{m}(B) \rightarrow G_{m}(\hat{B})\right] \rightarrow \operatorname{coker}\left[G_{m}\left(B\left[h^{-1}\right]\right)\right.$ $\left.\rightarrow G_{m}\left(\hat{B}\left[h^{-1}\right]\right)\right]$ is an isomorphism for any nonzero homogeneous element $h$ in $B$. Then the natural map $C \ell(A) \rightarrow C \ell(\hat{A})$ is an isomorphism. (See Lemma 1.3 (b) for situations in which part (ii) of the hypothesis is satisfied).

Proof. If $h_{1}$ in part (i) is not in $A$ we replace $h_{1}$ by its norm with respect to the action of $G$ on $B$, i.e., we may assume that $h_{1} \in A$. Since $A$ is a graded subring of $B$, the different is a homogeneous ideal and, moreover, its completion is the different for the ring extension $\hat{A} \rightarrow \hat{B}$. Hence there is a homogeneous element $h_{2}$ in $A$ so that $A\left[h_{2}^{-1}\right] \rightarrow B\left[h_{2}^{-1}\right]$ and $\hat{A}\left[h_{2}^{-1}\right] \rightarrow \hat{B}\left[h_{2}^{-1}\right]$ are separable extensions at prime ideals of height one. Now let $h=h_{1} h_{2}$. Then both ring extensions $A\left[h^{-1}\right] \rightarrow B\left[h^{-1}\right]$ and $\hat{A}\left[h^{-1}\right] \rightarrow \hat{B}\left[h^{-1}\right]$ are separable at prime ideals of height one, and both $B\left[h^{-1}\right]$ and $\hat{B}\left[h^{-1}\right]$ are factorial. From Galois descent we have the natural isomorphisms $C \ell\left(A\left[h^{-1}\right]\right) \stackrel{\sim}{\longrightarrow} H^{1}\left(G, G_{m}\left(\hat{B}\left[h^{-1}\right]\right)\right)$. Our assumption of 
part (ii) above and the argument in Corollary 1.4 now give that the map $C \ell\left(A\left[h^{-1}\right]\right) \rightarrow C \ell\left(\hat{A}\left[h^{-1}\right]\right)$ is an isomorphism (because the cohomology groups are isomorphic as in the proof of Corollary 1.4). Therefore we obtain the following commutative diagram

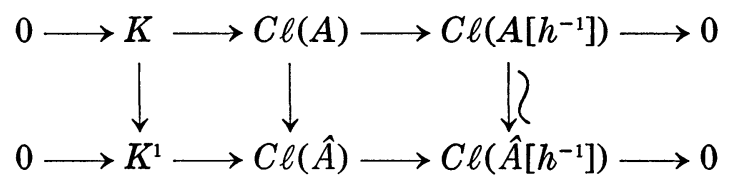

where $K$ and $K^{1}$ are generated by those classes in $C \ell(A)$ and $C \ell(\hat{A})$, respectively, that have a representative containing $h$. But Lemma 1.5 and Corollary 1.6 give that $K \rightarrow K^{1}$ is an isomorphism and consequently that $C \ell(A) \rightarrow C \ell(\hat{A})$ is an isomorphism.

\section{The main theorems}

We first need to introduce some further notation and definitions. Let $G$ be an affine, linearly reductive algebraic group and consider a linear representation $\rho: G \rightarrow G L(n, k)$. We denote by $X(G)$ the character group of $G$, that is, the morphisms $\chi: G \rightarrow k^{*}$. In this situation we have the induced action of $G$ on $R=k\left[X_{1}, \ldots, X_{n}\right]$ and $R^{G}$ is a finitely generated, normal $k$-algebra. We set $V_{x}=\{f \in R \mid g(f)=\chi(g) f\}$, that is, the semi invariants of $R$ of weight $\chi$. Because the action of $G$ on $R$ preserves the grading on $R$, it follows that $V_{\chi}$ is a graded $A=R^{G_{-}}$ module. Moreover, if $\hat{V}_{x}=\{f \in \hat{R} \mid g(f)=\chi(g) f\}$, one may easily observe that $\hat{V}_{x}=V_{x} \otimes_{A} \hat{A}$, since the action of $G$ preserves the homogeneous components of $\hat{R}$. Consequently, $V_{x} \neq 0$ if and only if $\hat{V}_{x} \neq 0$. If $V_{x}$ $\neq 0$ and if $f$ is a nonzero element of $V_{x}$, then we say that $\chi$ occurs for the representation $\rho$ if and only if both $V_{x}$ and $V_{x-1}$ are nonzero. We denote by $X(\rho, G)$ the set of characters of $G$ that occur for the representation $\rho$. Finally, we note that the preceding discussion remains valid when $R$ is replaced by a normal domain $B$, which is a finitely generated graded $k$-algebra and where the action of $G$ is linear on $B$.

LEMMA 2.1. Let $k$ be a field and let $B$ be a graded k-algebra that is a normal domain. We assume that $B$ is generated over $k$ by its forms of degree one. Suppose the linearly reductive group $G$ acts linearly on $B$, that is, the action of $G$ is induced by a representation 
$\rho: G \rightarrow G L(V)$, where $V$ denotes the vector space of forms of degree one. Put $A=B^{G}$.

(i) If $\chi$ occurs for $\rho$, then $V_{\chi}$ is isomorphic to an ideal of $A$. Similarily, $\hat{V}_{x}$ is isomorphic to an ideal of $\hat{A}$.

(ii) $X(\rho ; G)$ is a subgroup of $X(G)$.

(iii) If $f, d \in V_{x}$ are both nonzero, then the $\operatorname{map} \phi: B^{G} \cap(f B) \rightarrow B^{G}$ $\cap(d B)$ defined by $\phi(r)=(d / f) r$ is an $A=B^{G}$-isomorphism. Moreover, this statement remains true when $A$ and $B$ are replaced by their respective completions.

Proof. Suppose that $\chi$ occurs for $\rho$ and let $f$ be a nonzero element of $V_{\chi-1}$. Then the $A$-homomorphism $f \mapsto f f_{1}$ gives an embedding of $V_{\chi}$ into $A$. So (i) is established. Part (iii) is elementary. In regard to statement ii), the preceding discussion shows that $\chi$ occurs for $\rho$ if and only if $\chi^{-1}$ occurs. Moreover, it is elementary that $X(\rho ; G)$ is closed under products. Thus $X(\rho ; G)$ is a subgroup of $X(G)$.

LEMMA 2.2. Let the notation and hypothesis be the same as in Lemma 2.1. In addition, assume that $G$ is connected and that $\hat{B}$ is a factorial domain.

a). Let $\mathfrak{p}$ be a prime ideal of height one in $\hat{B}^{a}=\hat{A}$. Then there is a principal prime $f \in \hat{B}$ so that $\mathfrak{p}=\hat{B}^{G} \cap(f \hat{B})$.

b). For $f$ as in part a), there is a 1-cocycle $u: G \rightarrow G_{m}(\hat{B})$ such that $g(f)=u(g) f$ for $g \in G$.

Proof. Let $S=\hat{B}^{G}-\mathfrak{p}$. One easily checks that $\left(S^{-1} \hat{B}\right)^{G}=\left(\hat{B}^{G}\right)_{p}$ and that $S^{-1} \hat{B}$ is faithfully flat over the discrete valuation ring $\left(\hat{B}^{G}\right)_{\ddagger}$. Consequently, there is a prime element $f \in \hat{B}$ (recall that $\hat{B}$ is factorial) such that $\mathfrak{p}=\hat{B}^{G} \cap(f \hat{B})$. This establishes part a). Since $f$ necessarily divides an invariant, it follows that the orbit of $f$ under $G$ is finite (up to associates). Since $G$ is connected, we have that $g(f)=u(g) f$, where $u(g) \in G_{m}(\hat{B})$ for each $g \in G$. Finally, one easily checks that $u(g h)$ $=g(u(h)) u(g)$ for each $g, h$ in $G$, that is, $u: G \rightarrow G_{m}(\hat{B})$ is a 1-cocycle.

The following theorem due to A. Magid [M] is essential in the proof of our main result.

THEOREM 2.3 (A. Magid). Let $R$ be an affine normal domain over $k$ and let $G$ be a connected algebraic group over $k$ acting rationally on R. If $C \ell(R)$ is finitely generated, then $C \ell\left(R^{G}\right)$ is also. 
Proposition 2.4. Let $B$ be a normal domain that is finitely generated and graded over $k$, and assume that $B$ and $\hat{B}$ are factorial. We assume $B$ is generated by its one-forms). Suppose the connected, linearly reductive group $G$ is acting linearly on $B$ through a representation $\rho$ on the one-forms of $B$. Put $A=B^{G}$. Then the natural map $C \ell(A)$ $\rightarrow C \ell(\hat{A})$ is an isomorphism.

Proof. Let $p$ be a prime ideal of height one in $\hat{A}$. By Corollary 1.6, in order to demonstrate that $p$ is in the image of the map $C \ell(A)$ $\rightarrow C \ell(\hat{A})$ it suffices to show that $\mathfrak{p}$ is $\hat{A}$ isomorphic to a reflexive $A$ ideal that contains a nonzero homogeneous element of $A$.

Recalling Lemma 2.2, we obtain an element $f \in \hat{B}$ such that $\mathfrak{p}=\hat{A}$ $\cap f \hat{B}$ and such that $g(f)=u(g) f$, for $g \in G$, where $u: G \rightarrow G_{m}(\hat{B})$ is a one-cocycle. By Lemma 1.1, there is a character $\chi: G \rightarrow k^{*}$ and a unit $w \in \hat{B}$ such that $g(f)=\chi(g) w g\left(w^{-1} f\right)$ for $g \in G$. Setting $f_{1}=w f$ we obtain that $\mathfrak{p}=\hat{A} \cap f_{1} \hat{B}$ and that $g\left(f_{1}\right)=\chi(g) f_{1}$, for each $g \in G$. Since $\mathfrak{p}$ $\neq 0$ it follows that $V_{x}$ and $V_{x-1}$ are both nonzero, that is, $\chi \in X(\rho ; G)$. An easy consequence of the fact that $G$ preserves the grading on $B$ is that there are nonzero homogeneous elements $b$ and $c$ in $B$ such that $g(b)=\chi(g) b$ and $g(c)=\chi^{-1}(g) c$, for $g \in G$. By Lemma 2.1 (iii), there is an $\hat{A}$-isomorphism (noting $\mathfrak{p}=\hat{A} \cap f B$ ) $\mathfrak{p} \rightarrow \hat{A} \cap b \hat{B}=\mathfrak{a}$, and from above a contains the homogeneous element $b c$ in $A$.

We now establish our main result.

THEOREM 2.5. Let $k$ be an algebraically closed field and let $G$ be a linearly reductive, affine algebraic group acting linearly on $R=k\left[T_{1}, \cdots\right.$, $T_{n}$ ] through a representation $\rho$ on the one-forms of $R$. Put $A=R^{G}$. Then the natural map $C \ell(A) \rightarrow C \ell(\hat{A})$ is an isomorphism.

Proof. Let $G^{0}$ denote the connected component of $G$ and let $B=R^{G^{0}}$. By Proposition 2.4, the natural map $C \ell(B) \rightarrow C \ell(\hat{B})$ is an isomorphism. Moreover, $A=B^{H}$, where $H$ is a finite group acting linearly on the graded normal domain $B$ with the property that char $(k)$ does not divide the order of $H$. Since $A$ is a graded subring of the graded ring $B$ and since $C \ell(B) \cong C \ell(\hat{B})$ is a finitely generated group by Theorem 2.3, one can find a nonzero homogeneous form $f \in A$ such that $B\left[f^{-1}\right]$ and $\hat{B}\left[f^{-1}\right]$ are factorial (here we use the fact that the finite generating set of homogeneous primes for $C \ell(B) \stackrel{\sim}{\longrightarrow} C \ell(\hat{B})$ have nontrivial contrac- 
tion in $A$ ) and such that $A\left[f^{-1}\right] \rightarrow B\left[f^{-1}\right]$ and $\hat{A}\left[f^{-1}\right] \rightarrow \hat{B}\left[f^{-1}\right]$ are separable ring extensions. Thus by Lemma 1.3 (b) and Proposition 1.7, our result is established.

Remark. We would like to note here that Theorem 2.5 remains true if one replaces $R=k\left[T_{1}, \cdots, T_{n}\right]$ by a finitely generated, normal domain $B$ graded over $k$ with the properties that $\hat{B}$ (and hence also $B$ ) is factorial and Lemma 1.3 (b) is statisfied.

One case that still remains unsettled is that of a finite group $G$ acting linearly on $k\left[T_{1}, \cdots, T_{n}\right]=R$ under the assumption that char $k$ $=p$ divides $|G|$. If $u: G \rightarrow G_{m}(\hat{R})$ is a one cocycle in $H^{1}\left(G, G_{m}(\hat{R})\right)$ of order prime to $p$, it is possible to show that $u(g)=\chi(g) v(g)$ where $\chi$ is a character and $v(g)=w g\left(w^{-1}\right)$ for $w \in G_{m}(\hat{R})$. One uses the same technique as in Lemma 1.1, since the cohomology groups $H^{1}\left(G, R_{(i)}\right)$ are necessarily $p$-groups. One may deduce from this fact (as in Corollary 1.4 and Proposition 1.7) that it suffices to demonstrate that the $p$-component of $H^{1}\left(G, G_{m}(\hat{R})\right)$ is zero. However, since the $p$-component of $H^{1}\left(G, G_{m}(\hat{R})\right)$ is naturally an epimorphic image of $H^{1}\left(G_{p}, G_{m}(\hat{R})\right)$, where $G_{p}$ denotes the Sylow $p$-subgroup of $G$, it suffices to show that $H^{1}\left(G, G_{m}(\hat{R})\right)=0$ when $G$ is a $p$-group. Since $G$ has a normal series with $Z / p Z$ as the factors, one can obtain the desired result if one can show that $H^{1}\left(Z / p Z, G_{m}(\hat{B})\right)=0$ under the assumption that $B$ satisfies:

1) $B$ is a normal graded subring of $R=k\left[T_{1}, \cdots, T_{n}\right]$ such that $R$ is a finite integral extension of $B$.

2) $B$ and $\hat{B}$ are factorial.

3) $Z / p Z$ acts linearly on $B$.

Special cases of this result were settled in the affirmative in $[F-G]$ where the action of $Z / p^{n} Z$ on the one-forms of $k\left[T_{1}, \cdots, T_{p^{n}}\right]=R$ was via the regular representation of $Z / p^{n} Z$ on a $k$-vector space of dimension $p^{n}$. The technique in $[\mathrm{F}-\mathrm{G}]$ centered around an inverse limit argument after having computed the $G$-module structure on $R_{(i)}$ and the cohomology groups $H^{1}\left(G, R_{(i)}\right)$ (which need not be zero in this case). To suggest that the general problem is not completely trivial, we point out that Example 17.13 [F; page 102,103] (see also Danilov [D]) shows that the natural map $C \ell\left(R^{G}\right) \rightarrow C \ell\left(\hat{R}^{a}\right)$ is not in general an isomorphism when one replaces the $G$-action by that of derivations acting on $R$. 


\section{REFERENCES}

[B] M.-J. Bertin, Anneaux d'invariants d'anneaux de polynômes, en caractéristique p, C. R. Acad. Sc., Paris, t. 264, série A (1967), 653-656.

[D] V. I. Danilov, On a conjecture of Samuel,-Math. USSR-S610, 127-137 (1970) (Mat. sb. 81 (123), 132-144 (1970)).

[D-G] M. Demazure and P. Gabriel, Groupes Algebriques, North-Holland Publishing Co., Amsterdam, 1970.

[F] R. Fossum, The Divisor Class Group of a Krull Domain, Ergebnisse der Mathematik und ihrer Grenzgebiete, Vol. 74, Berlin-Heidelberg-New York, Springer, 1973.

[F-G] R. Fossum and P. Griffith, Complete local factorial rings which are not CohenMacaulay in characteristic $p$, Ann. Scient. Ec. Norm. Sup., $4^{\circ}$ serie, t. 8 (1975), 189-200.

[EGA] A. Grothendieck and J. Dieudonne, Elements de Geométric algébrique I.H.E.S. Publ. Math., Vol. I, no. 4, 1960.

[M] A. Magid, Finite generation of class groups of rings of invariants, Proc. A.M.S. 60 (1976), 45-48.

Illinois University 